\title{
NO RECURSIVELY ENUMERABLE SET IS THE UNION OF FINITELY MANY IMMUNE RETRACEABLE SETS ${ }^{1}$
}

\section{K. I. APPEL}

In what follows, by retracing function we will always mean special retracing function: a partial recursive function $f$ whose range is contained in its domain and which is associated with a unique point $b$ such that for each $x$ in the domain of $f, f(x) \leqq x$ and for some $n$, $f^{n}(x)=b$.

Suppose $R$ were a recursively enumerable set which was the union of $n$ immune retraceable sets with $n$ as small as possible. Then $R$ must be infinite by definition of immunity. Let $R=S_{1} \cup \ldots \cup S_{n}$ where each $S_{i}$ is retraced by partial recursive function $f_{i}$ to basepoint $b_{i}$. We observe that if $T$ is any infinite recursive subset of $R$, for each $i, T \cap S_{i}$ is immune retraceable. For let $B=\left\{b_{1}, b_{2}, \cdots, b_{n}\right\}$ and $T^{*}=T \cup B$. Then $T^{*}=S_{1}^{*} \cup \ldots \cup S_{n}^{*}$ where $S_{i}^{*}=T^{*} \cap S_{i}$. Now $S_{i}^{*}$ is retraceable for it is retraced by the function $f_{i}^{*}$ where domain $f_{i}^{*}=T$ $\cap \operatorname{Dom} f_{i}$ and $x \in \operatorname{Dom} f_{i}^{*} \Rightarrow f_{i}^{*}(x)$ is the largest element $y$ of $T^{*}$ such that for some $m f_{i}^{m}(x)=y$. Hence $S_{i}^{*}$ could only be immune or finite, but the minimality of $n$ insures that it must be immune. But $B$ is finite so our conclusion holds without adjunction of $B$.

Next, we use the previous observation to show that the intersection of the domains of the $f_{i}$ is infinite. For if this were not the case we could choose a largest subcollection of the $f_{i}$ whose domains had infinite intersection and enumerate the intersection of their domains. This infinite recursively enumerable subset of $R$ contains only finitely many members of the domain of some $f_{j}$ not in the subcollection hence only finitely many members of the corresponding $S_{j}$. But this set contains an infinite recursive subset with finite intersection with $S_{j}$ contradicting our observation.

Since the intersection of the domains of the $f_{i}$ is infinite recursively enumerable we may choose an infinite recursive subset of this intersection which (by our observation) is the union of $n$ immune retraceable sets; or equivalently, assume that $R$ is recursive and each $f_{i}$ has $R$ as domain.

If, for some $n, f_{i}^{n}(x)=y$ we will write $i: x \rightarrow y$. If this happens for no $n$, we write $i: x \rightarrow y$. We notice that if $y \notin S_{j}$ then $\{z \mid j: z \rightarrow y\}$ is finite. For if it were infinite, since if it is disjoint from $S_{j}$ by the defi-

Received by the editors March 10, 1966.

1 This generalizes the (unpublished) theorem of $\mathrm{R}$. Mansfield that $N$, the set of natural numbers, is not the union of two disjoint immune retraceable sets. 
nition of retracing function, it would have an infinite recursive subset disjoint from $S_{j}$, contradicting our observation. Also we note that since $n$ is minimal, for each $i, S_{i}-\bigcup_{j \neq i} S_{j}$ is infinite (for otherwise $\bigcup_{j \neq i} S_{j}$ would be a smaller example).

Now we propose to contradict the initial supposition in the following way. We will show that $R-S_{1}$ is recursively enumerable. Since it contains $S_{2}-\bigcup_{j \neq 2} S_{j}$ it is certainly infinite and hence, if recursively enumerable, contains an infinite recursive subset of $R$ disjoint from $S_{1}$, contradicting our basic observation.

Since $R$ is recursive, we may assume that $R$ is the set of natural numbers. We must now describe an enumeration of $\bar{S}_{1}$.

Let $Z_{m}=\{0,1, \cdots, m\}$. By an assignment of $Z_{m}$, we mean a function $h: Z_{m} \rightarrow\{i \mid i=1, \cdots, n\}$. (We think of $h$ as assigning $x$ to $S_{h(x)}$.) We say that an assignment $h$ on $Z_{m}$ is contradictory if for some triple $(x, y, i), m \geqq x>y, h(x)=h(y)=i$ but $i: x \rightarrow y$. Otherwise, $h$ is called consistent. It is evident that if $x \in S_{1}$ and $x<m$ then there is a consistent assignment on $Z_{m}$ with $h(x)=1$. We will show that if $x \notin S_{1}$ then there exists $M$ such that every assignment on $Z_{M}$ mapping $x$ to 1 is inconsistent. Hence an enumeration of $\bar{S}_{1}$ would consist in checking each pair $m, x$ to see whether there is any consistent assignment on $Z_{m}$ assigning $x$ to $S_{1}$ and, if not, enumerating $x$ in $\bar{S}_{1}$. (We need the fact that each $f_{i}$ is total to see that all inconsistent assignments are discovered.)

Now we consider a fixed assignment $h$ on $Z_{M}$ (M-fixed) such that for some $x \notin S_{1}, h(x)=1$. We will show that if $M$ is sufficiently large then $h$ is inconsistent. For reductio add absurdum, assume that $h$ is consistent. Since $\{z \mid 1: z \rightarrow x\}$ is finite and $S_{1}-\bigcup_{j \neq 1} S_{j}$ is infinite we may let $g_{2}$ be the smallest member of $S_{1}-U_{j \neq 1} S_{j}$ which is greater than $\max \{z \mid 1: z \rightarrow x\}$. If $h(x)=1$ and $h$ is consistent and $M>g_{2}$, then $h\left(g_{2}\right) \neq 1$ and $g_{2} \notin S_{h\left(g_{2}\right)}$. So we may choose $g_{3}$ as the smallest element of $S_{h\left(g_{2}\right)}$ $-\bigcup_{j \neq h\left(g_{2}\right)} S_{j}$ which exceeds

$$
\max \left(g_{2}, \max \left\{z \mid h\left(g_{2}\right): z \rightarrow g_{2}\right\}\right) .
$$

Now

$$
M>g_{3} \Rightarrow h\left(g_{3}\right) \neq 1, h\left(g_{3}\right) \neq h\left(g_{2}\right) \quad \text { and } \quad g_{3} \notin S_{h\left(o_{3}\right)} .
$$

Continuing this procedure we finally obtain a $g_{n+1}$ such that if $M>g_{n+1}, h\left(g_{n+1}\right)$ is distinct from the $n$ distinct integers $1, h\left(g_{2}\right), \ldots$, $h\left(g_{n}\right)$, contradicting the fact that the range of $h$ had cardinal $n$. Hence, such a contradictory $M$ must exist and the theorem is proved.

Although it is possible to obtain a more complicated version of this argument to handle regressive sets, this is unnecessary since T. G. 
McLaughlin [3] cleverly observed that if a recursively enumerable set is the union of finitely many immune regressive sets then it is the union of finitely many immune retraceable sets. Hence no recursively enumerable set is the union of finitely many immune regressive sets.

\section{REFERENCES}

1. K. I. Appel and T. G. McLaughlin, On properties of regressive sets, Trans. Amer. Math. Soc. 115 (1965), 83-93.

2. J. C. E. Dekker and J. Myhill, Retraceable sets, Canad. J. Math. 10 (1958), 357-373.

3. T. G. McLaughlin, Splitting and decomposition by regressive sets. II, Canad. J. Math. (to appear).

UNIVERSITY OF ILLINOIS 